\title{
Pseudoaneurysm with Candidal Infection after Renal Transplantation
}

\author{
Chee Hwee Lee ${ }^{1}$, Yu-Chien $\mathrm{Kao}^{2}$ and Wing P. Chan ${ }^{1,3}$
}

Key words: Infection, Kidney, Pseudoaneurysm, Transplantation

(Intern Med 50: 2679-2680, 2011)

(DOI: 10.2169/internalmedicine.50.6196)

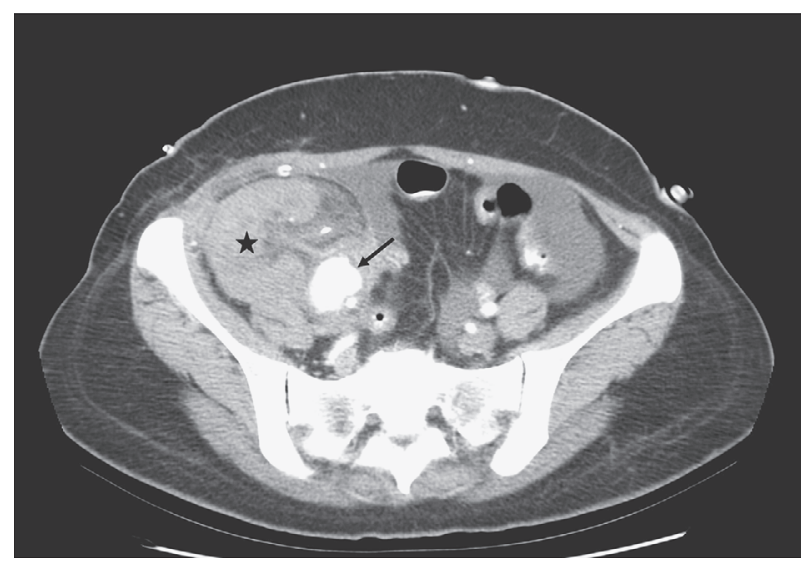

Picture 1.

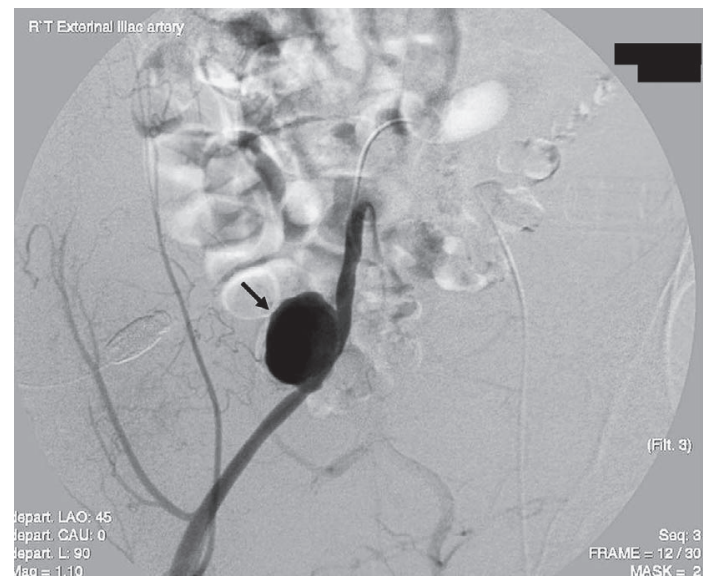

Picture 2.

A 30-year-old woman with end-stage renal disease secondary to glomerulonephritis was admitted to our hospital for

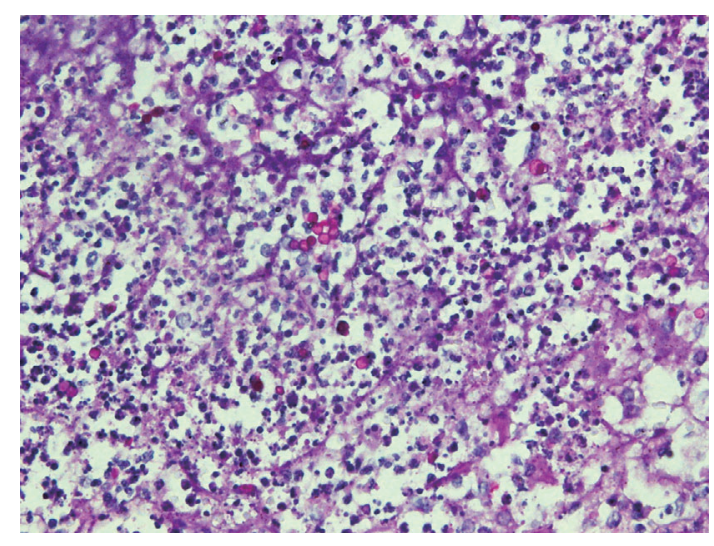

Picture 3.

kidney transplantation in the right iliac fossa. The urine output of the patient improved markedly after transplantation. Two months after transplantation, she was rehospitalized because of refractory urinary tract infection and decreasing urine output. Contrast-enhanced computed tomographic scan showed a pseudoanerysm (arrow) in the transplanted kidney (star) (Picture 1). Urine culture revealed a Candida colony. Catheter angiography was arranged for evaluation of the arterial supply to the graft; however, catheter angiography revealed poor opacification of the transplanted artery and a pseudoaneurysm (arrow) in the right external iliac artery (Picture 2). Unfortunately, due to deterioration of the graft condition, the patient underwent transplantectomy and repair of the right iliac artery. The final pathology examination with Periodic Acid-Schiff revealed Candidal infection with the presence of yeast with occasional budding and scanty pseudohyphae (Picture 3). Vascular complications are a significant cause of morbidity following a kidney transplant (1). Pseudoaneurysm, despite its rarity, is most com-

\footnotetext{
${ }^{1}$ Department of Radiology, Wan Fang Hospital, Taipei Medical University, Taiwan, Republic of China, ${ }^{2}$ Department of Pathology, School of Medicine, Taipei Medical University, Taiwan, Republic of China and ${ }^{3}$ Department of Radiology, School of Medicine, Taipei Medical University, Taiwan, Republic of China

Received for publication July 16, 2011; Accepted for publication August 23, 2011

Correspondence to Dr. Wing P. Chan, wingchan@tmu.edu.tw
} 
monly caused by Candida albicans and Staphylococcus aureus; however, multiple pathogens have been recorded (2).

The authors state that they have no Conflict of Interest (COI).

\section{References}

1. Tobben PJ, Zajko AB, Sumkin JH, et al. Pseudoaneurysms complicating organ transplantation: roles of CT, duplex sonography, and angiography. Radiology 169: 65-70, 1988.

2. Osman I, Barrero R, Leon E, Medina R, Torrubia F. Mycotic pseudoaneurysm following a kidney transplant: a case report and review of the literature. Pediatr Transplant 13: 615-619, 2009.

\footnotetext{
(C) 2011 The Japanese Society of Internal Medicine http://www.naika.or.jp/imindex.html
} 